\title{
The enigmatic role(s) of P2RY8-CRLF2
}

\section{Renate Panzer-Grümayer, Stefan Köhrer and Oskar A. Haas}

$C R L F 2$, the gene encoding the cytokine receptorlike factor 2, is located in the pseudoautosomal region 1 (PAR1) of both sex chromosomes. Its aberrant expression is the defining feature of an otherwise genetically heterogeneous relapse-prone group of $\mathrm{B}$ cell precursor acute lymphoblastic leukemias (BCP ALL) in children and adolescents. The two most common causative genetic defects are, first, an interstitial deletion that juxtaposes the first noncoding exon of $P 2 R Y 8$ to the entire CRLF2 coding region and, second, a chromosomal translocation that places $C R L F 2$ under the control of the $I G H$ enhancer [1]. IGH-CRLF2 fusions occur in a hematopoietic stem/ progenitor cell and are considered initiating events while P2RY8-CRLF2 fusions are caused by illegitimate V(D) $\mathrm{J}$-mediated recombination and therefore only occur in a $\mathrm{B}$ precursor cell. Both $C R L F 2$ fusions often carry additional alterations in CRLF2/IL7R/JAK-STAT pathway genes and may cooperatively activate downstream pathways. Interestingly, basically all $I G H-C R L F 2$ fusions belong to the Ph-like group of B-other ALL while only a smaller proportion of those with a $P 2 R Y 8-C R L F 2$ fusion do [2]. Considering these facts and additional differences in the ethnic background, age, sex, WBC and outcome of patients affected by the one or other fusion, it appears likely that these two fusions define distinct disease entities. They have a particular association with chromosome 21 alterations, either in the form of constitutional trisomy (half of all Down syndrome ALL have such a fusion) or, albeit exclusively for $P 2 R Y 8-C R L F 2$, in the form of a somatically acquired primary or secondary alteration.

Although $P 2 R Y 8-C R L F 2$ itself is a well-recognized risk indicator for relapse, its specific biological relevance for disease recurrence is still unclear and therefore a matter of ongoing discussion. Outcome data from different study groups are difficult to compare and interpret, because analyzed cohorts, patient numbers, observation periods and treatment regimens differ, as do screening techniques and ascertainment algorithms. To clarify these issues in a systematic way, we evaluated the salient features of P2RY8-CRLF2-positive leukemias by quantifying their clone sizes at diagnosis and relapse and correlated them with the respective $C R L F 2$ expression levels. These analyses revealed that the majority of $P 2 R Y 8-C R L F 2-$ positive clones are small at diagnosis and virtually never evolve into dominant relapse clones. These findings therefore prove that neither the presence of this alteration nor CRLF2 overexpression alone provide the affected clones with any noteworthy proliferative or selective advantage [3].

As mentioned before, P2RY8-CRLF2 often evolves as a secondary alteration in leukemias with preexisting, usually primary changes such as a iAMP21, hyperdiploidy and $\operatorname{dic}(9 ; 20)$. They concur with other deletions mainly affecting $B$ cell differentiation, cell cycle control and tumor suppression. With the exception of $P 2 R Y 8-C R L F 2$, these genetic alterations are generally conserved in the dominant relapse clone. To analyze the way in which these clones differ, we studied the mutation and expression patterns at diagnosis and relapse with whole exome and

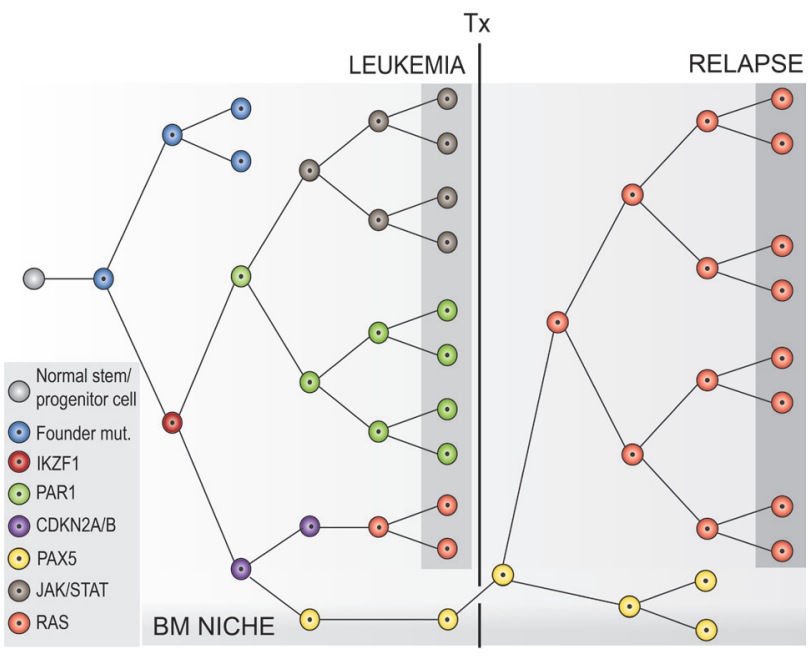

Figure 1: Model for the evolution of P2RY8-CRLF2positive leukemia and selection of relapse clones. Leukemia-initiating (founder) alterations generally occur in a hematopoietic stem/progenitor cell - and are present in all progenies, while the ensuing RAG-mediated microdeletions evolve only later during early $B$ cell differentiation. Microdeletions affect genes critical for B cell differentiation and tumor suppression. JAK/STAT and/or RTK/Ras pathway activating alterations may emerge during the same time span, are usually subclonal and do not outcompete each other at initial presentation of leukemia. Chemotherapy (Tx) then selects for resistant clones (e.g. IKZF1 mutant), which are either already present in the dominant clone, as depicted here, or in a small subclone of the initial leukemia. IKZFI mutant clones reside in the bone marrow (BM) niche and are there protected from chemotherapy. Only acquisition of proliferation-driving mutations leads to manifestations of the relapse. The model is based on the most striking results of NGS data from our latest study [4]. Each color in the figure represents a particular alteration in a cell and its descendent cells, in which further mutations occur (color code at left bottom of the graph). 
RNA sequencing in 41 relapsing and non-relapsing cases. Although all of them had a predominant P2RY8$C R L F 2$-positive clone at diagnosis, it reappeared in only 13 of $19(68 \%)$ investigated paired cases at relapse. $J A K / S T A T$ and $R T K / R A S$ alterations were present in $78 \%$ cases at diagnosis, appeared often sub-clonal, and coexisted together in multiple clones. Such mutations were present in $95 \%$ of relapse samples, but only half of the initial mutations were conserved at relapse. Thus, even though both $J A K / S T A T$ and $R T K / R A S$ pathway mutations apparently foster the emergence of disease recurrence in $P 2 R Y 8-C R L F 2$-positive leukemias, genetic alterations of $I K Z F 1$ are likely to be a more relevant factor in this context, because they already initially prevailed in relapsing cases and increased from 36 to $58 \%$ in matched samples. Moreover, these cases also had a typical IKZF1 transcriptional signature, reflecting a combination of stem cell properties, impaired lymphoid differentiation, enhanced focal adhesion, activation of the hypoxia pathway, deregulation of the cell cycle and drug resistance [4]. These characteristics become also apparent in mouse models in which Ikzfl-/- pro/pre-B cells acquire stem cell and adhesion properties including activation of the focal kinase pathway. They further mirror the features in a recent patient-derived xenograft murine model demonstrating the existence of a rare leukemic subpopulation that displays dormancy, stemness and treatment resistance properties as well as persistence in the bone marrow niche [5]. Besides epigenetic changes as likely causes for the plasticity of leukemic cells in generating this small "stem cell" pool, genetic alterations such as $I K Z F 1$ alterations may play a role in fostering such a phenotype (Figure 1).

Based on these insights, we envision P2RY8-CRLF2 as a "latent or mini driver" mutation $[6,7]$, a refined term for an earlier described subtype of "passenger" mutation [8]. Such mutations are commonly present in sub-clones, and even though they can positively influence cellular processes, they are not critical for it, but may sometimes substitute for a driver mutation. The success of therapeutically targeting such genes may, consequently, be limited. IKZFI alterations, on the other hand, are, especially in this context, attractive actionable targets. IKAROS signaling can be restored by, for instance, casein kinase-2 inhibitors, in case a functional wildtype $I K Z F 1$ allele is still present, or, by inhibition of the activated focal adhesion kinase pathway, a downstream pathway responsible for homing in the niche. Apart from conventional chemotherapy, these approaches could then also be combined with various currently already available JAK/STAT, Ras/MEK/ERK and PI3K/mTOR pathway inhibitors.
Renate Panzer-Grümayer: Leukemia Biology Group, Children's Cancer Research Institute, Vienna, Austria

Correspondence to: Renate Panzer-Grümayer, email renate.panzer@ccri.at

Keywords: childhood ALL; CRLF2-fusion; P2RY8-CRLF2; clonal evolution; IKZFI alterations.

Received: September 14, 2017

Published: October 26, 2017

\section{REFERENCES}

1. Mullighan CG, et al. Nat Genet. 2009; 41:1243-6.

2. Roberts KG, et al. N Engl J Med. 2014; 371:1005-15.

3. Morak M, et al. Blood. 2012; 120:5134-42.

4. Vesely C, et al. Leukemia. 2017; 31:1491-501.

5. Ebinger S, et al. Cancer Cell. 2016; 30:849-62.

6. Castro-Giner F, et al. Nat Rev Cancer. 2015; 15:680.

7. Nussinov R, et al. Curr Opin Struct Biol. 2015; 32:25-32.

8. Greaves M, et al. Nature. 2012; 481:306-13.

Copyright: Panzer-Grümayer et al. This is an open-access article distributed under the terms of the Creative Commons Attribution License 3.0 (CC BY 3.0), which permits unrestricted use, distribution, and reproduction in any medium, provided the original author and source are credited. 